\title{
INDEX OF REFRACTION AND DISPERSION WITH THE INTERFEROMETER.
}

\author{
By C. A. Proctor.
} determine the index of refraction of a dispersion medium by means of an interferometer it is necessary to introduce into one arm of the instrument a known thickness of the substance to be tested, and to count the number of fringes which cross the field while this change in the light path is actually occurring, or else to employ two or more light sources of different wave-length. For if we merely determine, by moving one of the mirrors, the shift of the white light fringes due to the substance, the results obtained will be incorrect owing to the shift due to dispersion. ${ }^{1}$

The usual laboratory method of determining the true value of this fringe shift may be briefly outlined as follows : ${ }^{2}$ A plate of uniform thickness of the substance, e. g., glass, whose index is to be determined, is cut in two, and one half is mounted in each arm of the interferometer so as to cover half the field. For this purpose the instrument is provided with two extra frames capable of rotation about vertical axes, one of them being equipped with a slow-motion attachment for rotating. The two pieces of glass are arranged to cover the same portion of the field of view and are set normal to the light paths, and the interferometer is adjusted for white light fringes. When these adjustments are made the fringes in the portion of the field covered by the glass will be continuous with those in the remainder of the field. One glass is now turned through a convenient angle. The other glass is then turned by means of the slow-motion attachment, the fringes of sodium light being counted as they cross the field until the white-light fringes again occupy their former position. The angle through which either glass turns is measured with the aid of a telescope and scale. If this angle is $i$, the fringe

${ }^{1}$ C. R. Mann, Manual of Adv. Optics, p. 37.

${ }^{2}$ Loc. cit., p. $6 \mathbf{r}$. 
count $2 N$, the thickness of the glass $t$, the index of the glass $\mu$, and the wave-length of sodium light $\lambda$, it may readily be shown that

$$
\mu=\frac{(t-N \lambda)(\mathrm{I}-\cos i)+\frac{N^{2} \lambda^{2}}{2 t}}{t(\mathrm{I}-\cos i)-N \lambda} .
$$

The term $N^{2} \lambda^{2} / 2 t$ is negligible.

If we now restore one glass to a position normal to the light path and move one of the mirrors until the white light fringes in the part of the field covered by the glasses once more occupy their former position, the number of sodium fringes crossing the field will be greater than $2 N$, the difference, which we will call $2 N^{\prime}$, depending upon the dispersion. If we assume the relation

we have

$$
\mu=A+\frac{B}{\lambda^{2}}
$$

$$
N^{\prime}=\frac{2 B t^{\prime}}{\lambda^{3}}
$$

In this expression $t^{\prime}$ is the thickness of glass introduced into the path by the rotation. In this way we can obtain both the index and the dispersion of our substance, using light of a single wavelength.

The objection to this method is that it demands a sample of glass for test in the form of two plane parallel plates of the same thickness, and a specially equipped interferometer. This makes it in many cases unavailable, either as a practical method of determining indices, or as a laboratory exercise.

It occurred to the writer that the above procedure might be considerably simplified, and that resort to the laboratory scrap pile should furnish materials to satisfactorily take the place of the slow motion attachment on the interferometer for rotating the glass. Accordingly a very rough micrometer screw with its nut was taken from a disused coefficient of expansion apparatus. The graduated disc was removed and in its place was put an arm of brass about Io $\mathrm{cm}$. long and $0.5 \mathrm{~cm}$. thick. The nut was then secured in a clamp mounted on a heavy laboratory support in such a way that, as nearly as could be judged by eye, the screw was vertical. A 
spherometer from which the legs and disk had been removed was held by another clamp on the rod of the same support in such a way that the screw was in a horizontal position with its point bearing against the arm attached to the vertical screw. This contact was maintained with the aid of a spiral spring. The glass to be tested was waxed to the lower end of the vertical screw, and a galvanometer mirror to the upper end. This device was placed so that the glass projected into one path of an interferometer far enough to cover the upper half of the field. It furnished a slow motion for turning the glass which gave complete satisfaction.

The method of experiment employed was as follows. From a fragment of plate glass two pieces were cut approximately $3 \times 3$ $\mathrm{cm}$. each, one of which was mounted on the slow-motion apparatus as described above. As is generally the case the surfaces of this glass were not parallel, so that when it was introduced into one arm of the interferometer the fringes disappeared from the field owing to the deviation of the light passing through the glass. This effect could of course be corrected by the readjustment of one of the mirrors but such a procedure would entail the loss of the fringes in the lower part of the field, and even if the surfaces were nearly enough parallel so that the fringes were visible in both portions of the field, they would be at a very low visibility in one if white light fringes were present in the other. As it is convenient, though by no means essential, to have the fringes in the lower part of the field for purposes of reference, the second piece of glass was mounted in the other arm so that it covered the same part of the the field as did the first. This glass was then turned in its own plane until the fringes were of the same width in both portions of the field. This adjustment is possible only when the lack of parallelism of the surfaces of the two squares of glass is nearly the same, a condition which will generally be fulfilled if they are adjoining portions of the same plate. The glass attached to the slowmotion device was then set normal to the light, i.e., in the position of minimum retardation, and the central fringe of the white-light system brought to a fiducial line in the field. The glass was then turned and the sodium fringes counted until any desired number had crossed the field. Following the notation used above we will 
[VoL. XXIV.

call this number $2 N$. The angle $i$ through which the glass turned was measured with a telescope and scale. The movable mirror was then shifted and the fringes counted until the central fringe in white light was again on the fiducial line. This count was larger than $2 N$ by a number, $2 N^{\prime}$ given by equation (2). That such must be the case appears from the following considerations: If we call the central white light fringe at the start that of order zero, the fringe on the fiducial line after the glass has been turned will be that of order $2 N$. If now we move the mirror till $2 \mathrm{~N}$ fringes have crossed the field in the opposite direction the fringe of order zero will have returned to its former position. It will, however, be no longer the center of the white light system because of the shift of that system due to dispersion. As this shift is always in such a direction as to increase the apparent retardation we shall accordingly have to move the mirror further until $2 N^{\prime}$ more fringes have crossed the field before the new central fringe, which is that of order $-2 N^{\prime}$, coincides with the fiducial line.

From $N, N^{\prime}, i$, and the thickness of the glass $t$, we can, with the help of equations (I) and (2), compute the index and the dispersion constants of the glass.

Two of the sources of possible error in the above method are worthy of special consideration. These are the lack of parallelism of the surfaces of the glass, and the determination of the angle through which the glass is rotated.

If the faces of the glass are not parallel an error may be introduced owing to the fact that as it is rotated the thickness introduced into the path will not be given by the simple relation on which equation (I) is based, and will be different in different parts of the field. Such a condition will be indicated by a continual change in the width of the fringes as the glass is rotated. This difficulty may be entirely eliminated by so mounting the glass that the line of intersection of its two faces shall be perpendicular to the axis of rotation. In the case of glass No. 2 measurements on which are given below (a fragment of a microscope slide) the general irregularity of the thickness was such that the fringes were very much distorted. However, as the pattern and its position in the field did not alter appreciably during a shift of 150 fringes it may be assumed 
that no error in the fringe count is introduced. Of course the thickness must be measured at the point where the fringe shift is observed.

The angle to be measured is the rotation of the glass about an axis in its own plane. If the actual axis is not vertical (we assume the light path to be horizontal) the angle determined with the telescope and scale may not be the one desired. This possibility may be guarded against by setting the scale parallel to the glass at the start, and tilting it until its image in the telescope shows no vertical displacement as the glass is turned. The determination of the scale distance is however a matter of some difficulty, and it is in this measurement that the greatest source of error seems to lie. In the apparatus used by the writer for example the absolute error may well have amounted to a half millimeter or even somewhat more. This might be bettered by having the axis of rotation of the glass more definitely marked.

To illustrate the foregoing some determinations of the index and dispersion constants of two samples of glass were made. No I was a piece of plate glass and No. 2 a fragment of a microscope slide. In each case the constants $A$ and $B$ were determined from observations on sodium fringes. As a check the index for the mercury and for the hydrogen line was computed from $A$ and $B$, and also directly determined using vacuum tubes as the light source. The thickness of the glass was measured with micrometer calipers. In the case of the readings on No. I with different scale distances the glass was re-set each time the scale was moved. The results are given in the appended Table.

The method above described may be applied with satisfactory results to samples of glass varying in thickness from $1.5 \mathrm{~mm}$. up. By using polarized light and properly mounting the plate under test it may also be adapted to the test of crystals of a like range of thickness. Another method, however, suggests itself which might be more conveniently used with crystals, and which is adapted to use with much thinner samples of the material. This may be outlined as follows :

The interferometer is adjusted for white-light fringes, and the lamina whose index is to be determined is introduced into one arm 
Glass No. 1. Thickness $=8.741 \mathrm{~mm} . \quad A=1.5102, B=50.5 \times 10^{-10}$.

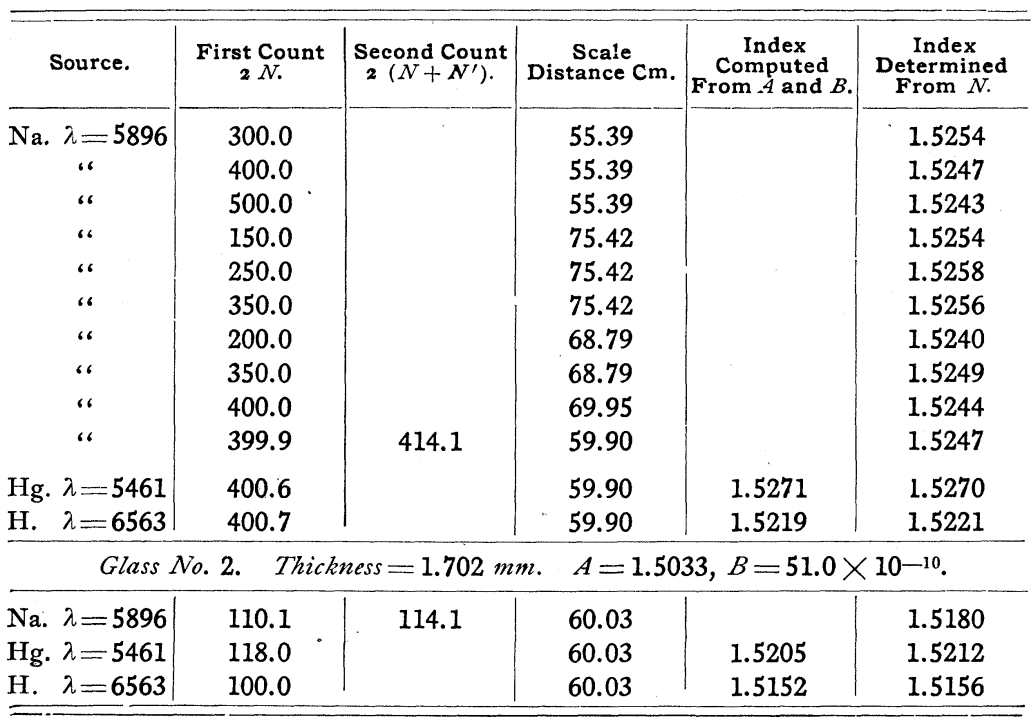

so that it covers a portion of the field and is normal to the light. One mirror is then moved till the white-light fringes return to their former position in the part of the field covered by the lamina, the fringes of sodium light which meantime cross the field being counted.

If $2 N_{1}=$ number of fringes counted,

$\mu_{1}=$ index of substance for sodium light,

$\lambda_{1}=$ wave-length of sodium light,

$B=$ second constant of Cauchy's formula,

$t=$ thickness of lamina,

we have the relation

$$
\left(\mu_{1}-\mathrm{I}\right) t=N_{1} \lambda_{1}-\frac{2 B t}{\lambda_{1}{ }^{2}}
$$

If we repeat this using light of wave-length $\lambda_{2}(e . g$., green mercury light) we shall have

$$
\left(\mu_{2}-\mathrm{I}\right) t=N_{2} \lambda_{2}-\frac{2 B t}{\lambda_{2}^{2}} .
$$

If now we attach the lamina to one of the mirror frames, we can obtain white-light fringes by reflection from either of its surfaces. 
If we count the number of sodium fringes $2 N_{3}$ which cross the field as we pass from one of these white-light systems to the other we shall have

$$
\mu_{1} t=N_{3} \lambda_{1}-\frac{2 B t}{\lambda_{1}^{2}} .
$$

$N_{3}$ must be corrected for the half wave-length change of phase occurring on reflection at the front surface.

From these three equations and Cauchy's formula the dispersion constants may be readily obtained.

UNIVERSITY OF MisSOURI, September 20, 1906. 